Rev Biomed 2000; 11:277-282.

\title{
Hemoparásitos en bovinos, caninos y equinos diagnosticados en el laboratorio de Parasitología de la Facultad de Medicina Veterinaria y Zootecnia de la Universidad Autónoma de Yucatan (1984-1999).
}

\section{Comunicación Breve}

Roger I. Rodríguez-Vivas, L.A. Cob-Galera, José L. Domínguez-Alpizar.

Universidad Autónoma de Yucatán. Facultad de Medicina Veterinaria y Zootecnia. Departamento de Parasitología. Mérida, Yucatán, México.

\section{RESUMEN.}

Introducción. Los hemoparásitos son organismos que pueden ser transmitidos a los animales domésticos por vectores mecánicos y biológicos. Su presencia en los animales domésticos produce cuadros hemáticos que afectan la salud animal.

Material y métodos. Se revisaron los archivos del laboratorio de Parasitología de la Facultad de Medicina Veterinaria y Zootecnia de la Universidad Autónoma de Yucatán, de marzo de 1984 a diciembre de 1999. Se obtuvo la información de las muestras sanguíneas de bovinos, caninos y equinos que fueron remitidas y procesadas mediante las técnicas de Knott y frotis sanguíneos teñidos con Giemsa al $10 \%$.

Resultados. Se analizaron un total de 3010 muestras sanguíneas, de las cuales 2438 fueron de bovinos, 493 de caninos y 79 de equinos. Los hemoparásitos que se diagnosticaron en las distintas especies animales fueron los siguientes: bovinos: Babesia bovis (2.78\%), Babesia bigemina (1.23\%) y Anaplasma marginale (15.79\%); caninos: Dirofilaria immitis (7.42\%), Dipetalonema reconditum (5.88\%) y Babesia canis $(3.92 \%)$, y equinos: Babesia equi (3.79\%) y Babesia caballi (2.53\%).

Conclusiones. Se concluye que los bovinos, caninos y equinos del estado de Yucatán se encuentran afectados por hemoparásitos que pueden afectar la salud y/o producción animal.

(Rev Biomed 2000; 11:277-282)

Palabras clave: Hemoparásitos, Babesia, Anaplasma, Dirofilaria, Dipetalonema, bovinos, caninos, equinos.

Solicitud de sobretiros: MSc. Roger I. Rodríguez-Vivas, Facultad de Medicina Veterinaria y Zootecnia, Universidad Autónoma de Yucatán. Departamento de Parasitología. Km 15.5 Carretera Mérida-Xmatkuil. AP 4-116D. Mérida, Yucatán, México. Fax. (99) 4232 05. E-mail: rvivas@tunku.uady.mx Recibido el 24/Marzo/2000. Aceptado para publicación el 2/Junio/2000. 
RI Rodríguez-Vivas, LA Cob-Galera, JL Domínguez Alpizar.

\section{SUMMARY.}

Haemoparasites in domestic animals, diagnosed in the Parasitology laboratory of the Facultad de Medicina Veterinaria y Zootecnia of the Universidad Autónoma de Yucatán (19841999).

Introduction. The haemoparasites are organisms that can be transmitted by mecanical and biological vectors to domestic animals. Their presence in domestic animals cause an haematic manifestation which affects animal health.

Material and methods. Data from the Parasitology laboratory of the Facultad de Medicina Veterinaria $y$ Zootecnia of the Universidad Autonoma de Yucatan, from March 1984 to December 1999, were analized. Information of blood samples from domestic animals tested by Knott technique and blood smear stained by Giemsa $10 \%$ were obtained.

Results. A total of 3010 blood samples were analized. The blood samples were from the following animal species: bovine 2438 , canine 493 , equine 79. The more frequent haemoparasites in different animal species were: bovine: Babesia bovis (2.78\%), Babesia bigemina (1.23\%) and Anaplasma marginale (15.79\%); canine: Dirofilaria immitis (7.42\%), Dipetalonema reconditum (5.88\%) and Babesia canis $(3.92 \%)$, and equine Babesia equi (3.79\%) and Babesia caballi $(2.53 \%)$.

Conclusion. It is concluded that bovine, canine and equine in the state of Yucatan are affected by haemoparasites that can affect animal health and production. (Rev Biomed 2000; 11:277-282)

Key words: Haemoparasites, Babesia, Anaplasma, Dirofilaria, Dipetalonema, bovine, canine, equine.

\section{INTRODUCCIÓN.}

Los animales domésticos se encuentran expuestos a numerosos microorganismos tales como bacterias, virus, rickettsias, mycoplasmas, clamidias, hongos, metazoarios y protozoarios. Los hemoparásitos agrupan una gran cantidad de agentes etiológicos causantes de enfermedades de gran trascendencia para la salud animal y salud pública a nivel mundial. Entre estos agentes encontramos un gran número de especies, principalmente rickettsias, nematodos y protozoarios (1). Los hemoparásitos pueden ser transmitidos a los animales domésticos por vectores mecánicos y biológicos. Su presencia en los animales domésticos produce cuadros hemáticos que afectan la salud animal.

La información generada en las investigaciones, hallazgos clínicos de campo, hallazgos en rastros, y reportes de clínicas y laboratorios, es de suma importancia en el diagnóstico de situación de las principales enfermedades en los animales domésticos (2). Esta información permite tener elementos para sentar las bases para el diseño de programas de prevención, control y erradicación de las enfermedades en diferentes regiones (3).

El objetivo del presente trabajo fue proporcionar información sobre la frecuencia de hemoparásitos diagnosticados en muestras sanguíneas enviadas al laboratorio de Parasitología de la Facultad de Medicina Veterinaria y Zootecnia de la Universidad Autónoma de Yucatán (FMVZUADY). El laboratorio de Parasitología de la FMVZ-UADY, se encuentra localizado en el municipio de Mérida, Yucatán, México.

El clima del Estado de Yucatán es tropical húmedo con lluvias en verano. La temperatura promedio anual fluctúa entre los $24.6 \mathrm{C}$ y $27.7 \mathrm{C}$ y la humedad relativa llega a $80 \%$ en la costa y $72 \%$ en el interior. La precipitación pluvial anual oscila entre $415 \mathrm{~mm}$ y $1290 \mathrm{~mm}$ (4).

\section{MATERIALES Y MÉTODOS.}

Para reportar los hemoparásitos diagnosticados en bovinos, caninos y equinos, se revisaron los archivos del laboratorio de Parasitología de la FMVZ-UADY, de marzo de 1984 a diciembre de 1999. Se obtuvo la información de las muestras

\section{Revista Biomédica}


Hemoparásitos en bovinos, caninos y equinos en Yucatán, México.

sanguíneas que fueron remitidas al laboratorio y procesadas mediante las técnicas de Knott y extendidos sanguíneos teñidos con Giemsa al 10\% descritas por Rodríguez et al. (5). Se registraron las siguientes variables: procedencia (Estado de Yucatán y otros Estados), especie animal (bovino, canino y equino) y tipo de parásito (especie y/o género). Las muestras remitidas al laboratorio provenían de animales de distinta raza, sexo, edad y condición corporal. Con esta información se elaboró una base de datos mediante el programa PANACEA (PAN Livestock Service, Reading University, U.K., Versión 1989) y se obtuvieron las frecuencias de los hemoparásitos respecto a cada especie animal, y la distribución de las muestras remitidas en relación a los años de muestreo y especie animal.

\section{RESULTADOS.}

De marzo de 1984 a diciembre de 1999 se analizaron en el laboratorio de Parasitolgía de la FMVZ-UADY un total de 3010 muestras sanguíneas de animales domésticos, de las cuales 2438 fueron de bovinos, 493 de caninos y 79 de equinos.

En el cuadro 1 se presenta la frecuencia de hemoparásitos diagnosticados en 2438 muestras sanguíneas de bovinos remitidas al laboratorio de Parasitología y procesadas mediante frotis sanguíneos teñidos con Giemsa al $10 \%$.

Cuadro 1

Frecuencia de hemoparásitos diagnosticados en 2438 muestras sanguíneas de bovinos remitidas al laboratorio de Parasitología de la FMVZ-UADY.

\begin{tabular}{lcc}
\hline $\begin{array}{l}\text { Tipo de } \\
\text { Parásito }\end{array}$ & Positivos & $\begin{array}{c}\text { Frecuencia } \\
(\boldsymbol{\%})\end{array}$ \\
\hline $\begin{array}{l}\text { Babesia bovis } \\
\text { Babesia bigemina }\end{array}$ & 68 & 2.78 \\
Anaplasma marginale & 30 & 1.23 \\
\hline
\end{tabular}

En el cuadro 2 se presenta la frecuencia de hemoparásitos diagnosticados en 493 muestras sanguíneas de caninos remitidas al laboratorio de Parasitología y procesadas mediante frotis sanguíneos teñidos con Giemsa al 10\% y la técnica de Knott modificada.

\section{Cuadro 2}

Frecuencia de hemoparásitos diagnosticados en 493 muestras sanguíneas de caninos remitidas al laboratorio de Parasitología de la FMVZ-UADY.

\begin{tabular}{|c|c|c|}
\hline $\begin{array}{l}\text { Tipo de } \\
\text { Parásito }\end{array}$ & Positivos & $\begin{array}{l}\text { Frecuencia } \\
(\%)\end{array}$ \\
\hline Dirofilaria immitis* & 29 & 7.42 \\
\hline $\begin{array}{l}\text { Dipetalonena } \\
\text { reconditum* }\end{array}$ & 23 & 5.88 \\
\hline Babesia canis** & 4 & 3.92 \\
\hline
\end{tabular}

* 391 muestras procesadas mediante la técnica de Knott modificada.

** 102 muestras procesadas mediante extendidos sanguíneos teñidos con Giemsa al $10 \%$.

En el cuadro 3 se presenta la frecuencia de hemoparásitos diagnosticados en 79 muestras sanguíneas de equinos remitidas al laboratorio de $\mathrm{Pa}$ rasitología y procesadas mediante frotis sanguíneos teñidos con Giemsa al 10\%.

\section{Cuadro 3}

Frecuencia de hemoparásitos diagnosticados en 79 muestras sanguíneas de equinos remitidas al laboratorio de Parasitología de la FMVZ-UADY.

\begin{tabular}{lcc}
\hline $\begin{array}{l}\text { Tipo de } \\
\text { Parásito }\end{array}$ & Positivos & $\begin{array}{c}\text { Frecuencia } \\
(\%)\end{array}$ \\
\hline $\begin{array}{l}\text { Babesia equi } \\
\text { Babesia caballi }\end{array}$ & 3 & $3.79 \%$ \\
\hline
\end{tabular}

De las 3010 muestras procesadas en el laboratorio de Parasitología, 2876 (95.55\%) provinieron de animales que habitaban en el estado de Yucatán, y 134 (4.45\%) procedieron de otros estados de la República Mexicana.

Vol. 11/No. 4/Octubre-Diciembre, 2000 


\section{RI Rodríguez-Vivas, LA Cob-Galera, JL Domínguez Alpizar.}

\section{DISCUSION.}

En las áreas tropicales y subtropicales de México las garrapatas y enfermedades que transmiten (babesiosis y anaplasmosis) causan importantes pérdidas económicas a la ganadería bovina (6). En Yucatán la garrapata Boophilus microplus es la responsable de la transmisión de Babesia bovis, Babesia bigemina y Anaplasma marginale a los bovinos. Cen (7) encontró que garrapatas $B$. microplus obtenidas de bovinos, presentan una tasa de infección con Babesia sp del $20.3 \%$ y concluyó que existe un proceso de adaptación entre $B$. $m i$ croplus y Babesia sp en nuestro medio. Se ha demostrado que existe una elevada prevalencia de reactores positivos y una estabilidad enzoótica bien definida para la anaplasmosis y babesiosis (8-13). La elevada población de garrapatas positivas a $B$. bovis y B. bigemina en nuestra región (7) ha permitido que la mayoría de los bovinos produzcan anticuerpos contra estos agentes, lo que explica la baja frecuencia de casos positivos a $B$. bovis y $B$. bigemina diagnosticados a partir de frotis sanguíneos. A. marginale se presentó con mayor frecuencia $(15.79 \%)$, probablemente debido al modo de transmisión de este agente. En el estado de Yucatán, México, A. marginale es transmitida a los bovinos tanto por garrapatas como por moscas hematófagas y/o instrumentos veterinarios conteniendo sangre contaminada, pudiendo con esto facilitar su transmisión. Dentro del grupo del orden Diptera que transmiten $A$. marginale en nuestra región destacan Tabanus sp, Stomoxys calcitrans y Haematobia irritans $(14,15)$.

La presentación de D. immitis en caninos de una región depende de factores climáticos que afectan la biología de los mosquitos (16). La capacidad de los mosquitos para transmitir el agente es variable dependiendo de la región; por ejemplo: Aedes vexans es un mosquito con un amplio potencial de trasmisión en la región centronorte de los Estados Unidos (17); sin embargo, no tiene un papel importante en el sureste (18). La capacidad de transmisión de los mosquitos es proporcional a la capacidad de vuelo (16).
Rodríguez et al. (19) encontraron en perros callejeros de la ciudad de Mérida, Yucatán, una frecuencia a D. immitis y D. reconditum de $6.54 \%$ y $7.47 \%$ respectivamente. Estas frecuencias fueron similares a las diagnosticadas en el laboratorio de Parasitología, a pesar de tratarse generalmente de animales de domicilio.

B. canis es un organismo intracelular transmitido por garrapatas y causa anemia hemolítica en los perros $(20,21)$. La garrapata café del perro (Rhipicephalus sanguineus) es el vector potencial de la babesiosis canina (22). Debido a que $R$. sanguineus puede trasmitir otros microorganismos, los perros pueden estar infectados de forma concomitante con hemoparásitos tales como B. canis, Ehrlichia canis y Hepatozoon canis (23), pudiendo manifestar signología clínica incluso hasta tres años posteriores a la infección por garrapatas $(24,25$, 26); sin embargo, hasta la fecha en nuestro laboratorio no se ha diagnosticado la presencia de $E$. canis y $H$. canis en perros del estado de Yucatán, México. A pesar de que $R$. sanguineus parasita en gran proporción a los perros de nuestra región, existen pocos casos reportados de E. canis. Esto pone de manifiesto la necesidad de difundir la importancia de esta enfermedad en la región y de realizar estudios más precisos.

Rodríguez y col. (27) reportaron que B. equi y B. caballi se encuentran presentes en equinos del estado de Yucatán, México, y hoy en día la babesiosis equina representa un problema importante. La baja frecuencia de casos positivos a Babesia sp en equinos, probablemente se debe al poco conocimiento de esta enfermedad por parte de los productores y veterinarios de campo, lo que se refleja en el bajo número de muestras (79) que fueron enviadas al laboratorio para su análisis. En el estado, existe una gran variedad de garrapatas potencialmente vectoras de estos agentes tales como Anocentor nitens y $R$. sanguineus (28), lo que implica un alto riesgo de transmisión de Babesia sp a los equinos.

Se concluye que los bovinos, caninos y equinos del estado de Yucatán se encuentran

\section{Revista Biomédica}


Hemoparásitos en bovinos, caninos y equinos en Yucatán, México.

afectados por hemoparásitos que pueden representar un problema de salud y producción animal.

\section{REFERENCIAS.}

1. Soulsby EJL. Parasitología y Enfermedades Parasitarias en los Animales Domésticos. 7a. ed. México: Interamericana; 1987. p. 729-39.

2. Blood DC, Henderson JA, Radostitis OM. Medicina Veterinaria. México: Interamericana; 1988. p. 1411.

3. Thrusfield M. Veterinary epidemiology, $2^{\text {nd }}$ edition Oxford: Blackwell Science; 1995. p.129-42.

4. Instituto Nacional de Estadística, Geografía e Informática. Gobierno del Estado de Yucatán. Aspectos geográficos. México: Anuario estadístico del estado de Yucatán. 1996. p.444.

5. Rodríguez VRI, Domínguez AJL, Cob GLA. Técnicas Parasitológicas en Medicina Veterinaria. Mérida: Universidad Autónoma de Yucatán; 1994. p.178-91.

6. Vega MA Current importance of cattle hemoparasite diseases. Segundo Seminario Internacional de Parasitología Animal, Garrapatas y Enfermedades que Transmiten. Octubre 9-11, Oaxtepec, Morelos. SAGAR; 1991. p.144-50.

7. Cen AF, Rodríguez VRI, Domínguez AJL, Wagner G. Studies on the effect of infection by Babesia sp on oviposition of Boophilus microplus engorged females naturally infected in the Mexican tropics. Vet Parasitol 1998; 78:253-7.

8. Ramos A, Alvarez M, Figueroa, V Solís, J, Rodríguez I, Hernández R, et al. Mem Ins Oswaldo Cruz, 1992; 87 (Suppl): 213-7.

9. Ramírez GT, Jones, TW, Brown CGD, Dominguez AJL, Honhold N. Bovine babesiosis in dual purpose calves in the state of Yucatan, Mexico. Trop An Hlth Prod 1998; 30:45-52.

10. Solís CJ, Rodríguez VRI, Dájer, AA. Presencia de anticuerpos contra Anaplasma marginale en becerros de 3 ranchos del oriente de Yucatán, México. Rev Biomed 1996; 7:19-25.

11. Solís CJJ, Rodríguez VRI, Dájer AA. Monitoreo de IgG e IgM a Babesia bigemina (Haemosporidia: Babesiidae) en becerros del trópico mexicano. Rev Biol Trop 1998; 46:1123-8.

12. Solorio RJ, Rodríguez VRI, Pérez E, Wagner G. Management factors associated with Babesia bovis seroprevalence in cattle from eastern Yucatan, Mexico. Prev Vet Med 1999; 40:261-9.

14. Wagner G, Cruz D, Holman J, Waghela S. Epidemiology, diagnosis and control alternatives for anaplasmosis. Segundo Seminario Internacional de Parasitología Animal. Garrapatas y enfermedades que transmiten. Morelos, México: SAGAR; 1991. p. 161-71.

15. Rodríguez, V.R.I.; Domínguez, A.J.L. Grupos entomológicos de importancia veterinaria en Yucatán, México. Rev. Bioméd. 1997; 9:26-37.

17. Hendrix CM, Bemrick WJ, Schlotthauer JC. Natural transmission of Dirofilaria immitis by Aedes vexans. Am J Vet Res 1980; 41:1253-5.

16. Knight DH. Heartworm infection. Veterinary Clinics of North America: Small Animal Practice 1987; 17:1463-518.

13. Mata MY. Determinación de factores de riesgo de manejo sobre la seroprevalencia de Anaplasma marginale en la zona ganadera oriente del estado de Yucatán, México. Tesis de Maestría. FMVZ-UADY. 1996 pp 73.

18. Otto GF, Jachowski LA. Mosquitos in canine heartworm disease In: Proceedings of the haertworm symposium 80 . Otto GF ed. Edwardsville, KS: Veterinary Medicine Publishing Co; 1981. p.17-32.

19. Rodríguez VRI, Cen AF, Domínguez AJL, Cob, GLA, Solís CJJ. Detección de espiroquetas del género Borrelia en hemolinfas de teleoginas de Boophilus microplus en el estado de Yucatán, México. Veterinaria México 1996; 27:187-8.

20. Pyle RL. Canine ehrlichiosis . J Am. Vet Med Assoc 1980; 177:1197-9.

21. Purnell RE. Babesiosis in various hosts. In: M. Ristic and J.P. Kreier ed. Babesiosis. New York: Academic Press; 1981. p. 25-63.

22. Harrus S, Bark H, Waner T. Canine monocytic ehrlichiosis: An update. Parasitol 1997; 19:431-44.

Vol. 11/No. 4/Octubre-Diciembre, 2000 
RI Rodríguez-Vivas, LA Cob-Galera, JL Domínguez Alpizar.

23. Troy GC, Forrester SD. Canine ehrlichiosis: Ehrlichia canis, E. equi and E. risticci infections. In: Greene, W.B., Ed. Infectious diseases of the dog and cat. Philadelphia: W.B. Saunders, Co; 1990, p.404-14.

24. Codner E C, Farris L. Characterization of the subclinical phase of ehrlichiosis in dogs. J Am Vet Assoc 1986; 189:4750 .

25. Lobetti RG. Canine babesiosis. Parasitol 1998; 20:41830.

26. Navarrete I, Nieto LCG. Babesiosis, hepatozoonosis y citauxzoonosis felina. En: Parasitología Veterinaria. Cordero CM, Rojo VFA ed. Madrid: McGraw-Hill. Interamericana; 1999. p. 672-8.

27. Rodríguez VRI, Domíngez AJL, Dzul CU, Ramos GI. Reporte de casos del género Babesia en equinos del estado de Yucatán, México. Univ y Cienc 1997; 12:123-8.

28. Woodham C, González O, Lopez L, Guereña M. Progresos en la erradicación de las garrapatas Boophilus en México 1960-1980. Rev Mund Zoot 1983; 48:18-24. 